\title{
Socio-demographic Profile and Drug Sensitivity Pattern of Suspected Drug Resistant Tuberculosis among Patients in Regional Tuberculosis Reference Laboratory (R.T.R.L) of a Tertiary Hospital
}

\author{
UMMAY FATEMA KHATUN, ${ }^{1}$ ROBED AMIN, ${ }^{2}$ MUNA ISLAM, ${ }^{3}$ ABDUR ROB,${ }^{4}$ ABDUR RAHIM ${ }^{3}$
}

\begin{abstract}
:
Background: Drug resistant tuberculosis has been reported in all regions of the world. In this study we address the socio-demographic profile and drug sensitivity pattern as well as prevalence of drug resistance tuberculosis in a tertiary center (regional TB reference laboratory) in Bangladesh.

Method: This Study was carried out in R.T.R.L. (RegionalTB Reference Laboratory) in 250 bedded Chittagong General Hospital. Patients who were referred to R.T.R.L during the period July 2012 to July 2013 were included in the study. Total 100 patients with suspected drug resistant tuberculosis (TB) who had any one of 9 criteria of NTP (National Tuberculosis Control Programme) were selected consecutively. Gene xpert MTB/RIF (Rifampicin resistance) test for all sputum positive cases were performed. Sputum sample of Patients with positive microscopy for AFB or positive Xpert/MTB was sent for culture. The samples with positive sputum culture were sent for drug sensitivity test for $I^{\text {st }}$ line anti- tubercular drug.

Result: Among 100 patients 78 were male and 22 were female, majority of the patients (64) were between $15-45$ years with poor socio economic condition (73\%) and primarily educated.Analysis of our Study result showed that $18 \%$ of patients were mono-drug resistant.Among them $13 \%$ showed resistance to isoniazid (INH), $4 \%$ to streptomycin and only $2 \%$ to rifampicin. No patient was found resistant to pyrazinamide (PNZ) and $38 \%$ of patient with suspected drug - resistant TB was found to have no drug resistance. $18 \%$ of patient had multidrug resistant tuberculosis (MDR-TB) among which $56 \%$ were relapse cases (48\% after cat $-1,8 \%$ after cat II), $24 \%$ were non - converter of cat $-I, I 2 \%$ belonged to failure of cat $-I, 3 \%$ failure of cat $-I I, 2 \%$ return after default and others. $I \%$ of patient had history of contact with MDR -TB patient.

Conclusion: Drug-resistance tuberculosis especially MDR-TB, was higher in patients with previously incomplete anti-tuberculosis treatment. $A$ high level of drug resistance among the re-treatment TB patients poses a threat of transmission of resistant strains to susceptible persons in the community. For this reason proper counseling of patients and attention towards the completion of the anti-TB treatment are needed.
\end{abstract}

Key words: MDR-TB, Drug resistant tuberculosis, Tuberculosis, RTRL.

\section{Introduction:}

With almost 9 million new cases each year, tuberculosis (TB) remains one of the most feared diseases on the planet. Led by the STOP-TB partnership and WHO, recent efforts to combat the disease have made considerable progress in a number of countries. Control of tuberculosis (TB) remains one of the most serious challenges to global health. However

1. Assistant Professor, Medicine, Chittagong Medical College Hospital, Chittagong, Bangladesh.

2. Associate Professor, Medicine, Dhaka Medical College Hospital, Dhaka, Bangladesh.

3. Junior Consultant, Medicine, 250 beded Chittagong General Hospital, Chittagong, Bangladesh.

4. Senior Consultant, Medicine, 250 beded Chittagong General Hospital, Chittagong, Bangladesh.

Corresponding author: Dr. Ummay Fatema Khatun, Assistant Professor, Medicine, Chittagong Medical College Hospital, Chittagong, Bangladesh. Contact Number: +880-1711199491, Email: ufk94@yahoo.com. the emergence of mutated strains of Mycobacterium Tuberculosis that are resistant to the major anti-tuberculosis drugs poses a deadly threat to control efforts. TB is predominantly a disease of poverty with over $80 \%$ of cases occurring in Asia and Africa. Although the greatest numbers of patients live in highly populous countries of Asia the highest incidence of disease is found in the WHO region of Africa. ${ }^{1}$

A new and potentially devastating threat to TB control is the emergence of strains that cannot be cured by standard antituberculous drug regimens. Drug resistance tuberculosis is defined as resistance to at least one Anti- tuberculosis drugs of $1^{\text {st }}$ line. Resistance to at least two major anti-tuberculous drugs, isoniazid and rifampicin has been termed as multidrug resistant tuberculosis (MDR-TB). ${ }^{2}$ Extensively drug resistant tuberculosis (XDR-TB) is a form of TB caused by bacteria that are resistant to isoniazid and rifampicin as well as any fluoroquinolone and any of the second- line anti- tuberculous 
injectable agents (amikacin, kanamycin and/ or capreomycin). ${ }^{2}$

MDR-TB results from either primary infection with resistant bacteria or may develop during the course of treatment. Drugresistance TB usually develops during treatment of infection with fully sensitive mycobacterium tubercular strain when the course of antibiotics is interrupted and concentrations of active drugs in the body are insufficient to kill $100 \%$ of bacteria. This can happen for a number of reasons. Patients may feel better and halt their antibiotic course, scarcity of drug supplies, or patients may forget to take their medication from time to time. Although mutations that cause resistance to certain drugs may also cause lower fitness in the mutated M. tuberculosis strains, selected mutation sites may allow MDR M. tuberculosis strains to spread from person to person as readily as drug-sensitive TB and in the same manner. ${ }^{3}$

The treatment of patients infected with MDR-TB strains is more complex, less effective, more toxic and more expensive than the treatment of patients infected by susceptible strains. ${ }^{4}$ These forms of TB do not respond to the standard six month treatment with first line anti- TB drugs and can take up to two years or more to treat with drugs. Control of drug resistant tuberculosis requires a strong health infrastructure to ensure the delivery of effective therapy coupled with surveillance and monitoring activities to enable timely intervention to limit transmission and spread of the disease. Although studies demonstrating successful treatment outcomes for MDR-TB cases have been reported from a number of settings, ${ }^{5}$ the allocation of resources to detect and treat MDR TB in poor resource settings remains controversial. ${ }^{6}$

Bangladesh is a highly populous country of low income group and ranked seventh in the list of 27 priority countries for MDR/XDR-TB. ${ }^{1}$ Our health infrastructures are poor and poor socio-economic condition is a strong risk factor for tuberculosis but their role in development of drug-resistant tuberculosis is not clear. In 2008, the World Health Organization (WHO) estimated that 440000 cases of MDRTB emerged globally and $85 \%$ of its global burden occurs in 27 countries. ${ }^{1}$ The extent of anti-TB drug resistance in Bangladesh is not well known. No nation-wide drugresistance survey was conducted. However limited survey was conducted by the Damien foundation and the international centre for Diarrhoeal Diseases Research, Bangladesh (ICDDRB) showed an overall prevalence rate of $2 \%-5.5 \%$. The rate of MDR-TB varied between 5.6\% and $15.4 \%$ in person who had received treatment for 1 month or more. The level of drug resistance is considered as an epidemiological indicator to assess the achievement of the
NTP, as well as it measures the extent of resistant bacteria prevailing in the community. Another community based survey in a rural urban set up reported that among the identified TB cases about $48.4 \%$ of M. tubercular isolates were resistant to at least one of the first line anti-tubercular drugs while multidrug-resistance was observed in $5.5 \%$ of isolates. ${ }^{7}$ A recent estimation made by WHO showed approximately $2.2 \%$ of new case and $14.7 \%$ of previously treated patients suffer from MDR-TB in Bangladesh. ${ }^{8}$ The extent of XDR-TB remain unknown in the absence of drugsusceptibility test (DST) for second-line anti-tubercular drugs. At the ministerial meeting of high MDR-TB burden countries (MDR-HBC) held in Beijing, China, In April 2009, Countries Committed to tackle the epidemic with innovation and urgency. In May 2009, the $62^{\text {nd }}$ World Health Assembly urged member states to achieve universal access to diagnosis and treatment of M/XDR-TB. By October 2009, 27 MDRHBCS (multidrug resistant - High burden countries) had began to update their national TB control plans to include a component on drug resistant TB in compliance with the resolution.

MDR or XDR- TB case finding unlike routine TB case finding requires advanced, costly and needs intensive laboratory technology. However measuring the magnitude and trends in the M/XDR- TB epidemic is essential for planning appropriate responses and assessing, their epidemiological impact on the response being implemented by countries. India, with second highest burden of MDRTB has achieved major progress in expanding laboratory capacity and is hailed as an example of the program being made globally. Uninterrupted and timely supply of medicines that meet international standards of quality assurance is an essential component of the response to X/ MDR-TB.

Although, most of the programmes treating MDR /XDRTB experience a variety of problems to guarantee access to this essential components. Un- interrupted and timely supply of medicines that meet international standards of quality assurance is an essential component of the response to XDR/ MDR-TB. The target of universal access to diagnosis and treatment of MDR-TB by 2015 set by the $62^{\text {nd }}$ world Health Assembly is ambitious. It requires countries to mobilize resources, build capacity and properly coordinate operations within the health care system.

To our knowledge, few other studies have specifically assessed socio-demographic profile and drug sensitivity pattern associated with drug resistant tuberculosis. The aim of this study was to identify risk factors, socio-demographic status and drug sensitivity associated with drug resistant tuberculosis as well as Prevalence of mono and multi - drug 
resistant Tuberculosis among patients referred to R.T.R.L in Chittagong General Hospital.

\section{Materials and Methods:}

This Study was carried out in R.T.R.L. (regional TB reference laboratory) in 250 bedded Chittagong General Hospital. Total 100 patients were selected consecutively. Patients who were referred to (R.T.R.L) In Ctg. general Hospital during the period July 2012 to July 2013 were included. Patients with suspected drug resistant tuberculosis (TB) and age 15 years or above who fulfill one of 9 criteria of NTP (National Tuberculosis Control Programme of Bangladesh) are included in this study. ${ }^{9}$ These Criteria are as follows:

i) Failures of category I (remain positive at month 5 or later and smear negative patients. Who become smear positive at months 2).

ii) Failures of Category II (remain positive at month 5 or 8).

iii) Non-converters of Category I (remain positive at month 3).

iv) Non-converters of Category II (remain positive at month 4).

v) All relapses (Category I and Category II).

vi) All return after default (Category I and Category II).

vii) Close contacts of MDR-TB patient with symptoms.

viii) All TB/HIV infected patients at the start of TB therapy.

ix) Others (History of irregular treatment from Private or Public sectors etc).

A prepared Data sheet is fulfilled for each patient. Ethical clearance is taken from each patient before interviewing. The study was approved by ethical committee of Chittagong General Hospital, Anderkilla, Chittagong. The variable analyzed included age, sex, occupation, residence, socio economic status, Previous history of contact with MDR TB patient, history of previous treatment for TB (defined as having received treatment with Anti-tuberculous drugs for more than 1 months.

In all suspected case of drug resistant TB a complete history was taken, physical examination were done and routine investigation such as complete blood count, X-ray chest P/ A view sputum for AFB (acid fast bacilli) by microscopy on two sample of sputum were carried out. Gene xpert MTB/ RIF (Rifampicin resistance) test was conducted for all patients. In patient with positive microscopy for AFB and or positive Xpert/MTB sputum sample was sent for culture. Positive Sputum samples were sent for drug sensitivity test for $1^{\text {st }}$ line anti- tubercular drug. Sputum was considered culture negative when no visible mycobacterial colony was grown on either of the Lowenstein Jensen (L-J) slants within 8 weeks of incubation at $37^{\circ} \mathrm{C}$. We did not assess blood sugar level, HIV tests, immune status of the patients and so this is our limitation of the study.

\section{Result:}

During the study period a total of 100 smear positive PTB patients were enrolled. $75 \%$ had positive culture and drug sensitivity test as done. Male to female ratio was 3.5:1. The mean age was 35 years (range: 15-65). Majority (64\%) of the patients belonged to $15-45$ years indicating that earning members of family are commonly affected. $62 \%$ of patients were primarily educated whereas $15 \%$ of patients were illiterate. Majority of patients were daily worker (36\%) and garments worker (35\%). Most of the affected patients belonged to low income group 73 (upto 80000 BDT per annum). The study showed $43 \%$ of patients live in rural area followed by $41 \%$ in slum area and $16 \%$ in urban area. Most of the patients (97\%) had previous history of treatment with anti-tubercular drug. No patient had history of contact with MDR -TB patient (Table I).

19 patients were found mono-drug resistant. Among them $13(68.42 \%)$ showed resistance to isoniazid (INH), 4 (21\%) to streptomycin and only $2(10.52 \%)$ to rifampicin. 38 patients was found to have no drug resistance (Table II).

Out of 18 MDR-TB patients 10 were relapse cases ( 8 after cat -I, 2 after cat II), 3 were non - converter of cat -I, 1 was non - converter of cat II, 2 patients belong to treatment failure, and 2 patients were return after default and others. No patients were found to have HIV and TB co-infection, or failure of cat II, or history of contact with MDR-TB. Among mono-drug resistance cases 14 patients were all relapse case (12 after cat -I, 2 after cat II), 1 patients were non - converter of cat-I, 3 patients were belongs to failure of cat -I, 1 patients were return after default. Alarmingly 5 patients out of $18 \mathrm{MDR}-\mathrm{TB}$ patients $(27.77 \%)$ isolates were found to be resistant to four anti-TB drugs (Isoniazide, Rifampicin, Ethambutol and Streptomycin). MDR-TB was significantly higher among the patients with history of antiTB treatment previously. MDR-TB was also high among males (75\%) and in patients with age below 45 years (76\%) (Table III). 
Table I

Socio-demographic characteristics of patients having suspected drug resistant-TB $(n=100)$

\begin{tabular}{|c|c|c|}
\hline Characteristics & Subcategory & Number \\
\hline \multirow[t]{2}{*}{ Age (yrs) } & $15-45$ years & 64 \\
\hline & 46-76 years & 36 \\
\hline \multirow[t]{2}{*}{ Sex } & Male & 78 \\
\hline & Female & 22 \\
\hline \multirow[t]{4}{*}{ Educational Status } & Illiterate & 15 \\
\hline & Up to primary & 62 \\
\hline & Secondary & 20 \\
\hline & HSC and above & 3 \\
\hline \multirow[t]{5}{*}{ Occupation } & Daily worker & 36 \\
\hline & Service holder (Garments worker) & 35 \\
\hline & Business & 12 \\
\hline & Retired & 15 \\
\hline & House wife & 2 \\
\hline \multirow[t]{3}{*}{ Locality } & Rural & 43 \\
\hline & Urban & 16 \\
\hline & Slum area & 41 \\
\hline \multirow[t]{3}{*}{ Monthly income } & Up to 80000 BDT per annum & 73 \\
\hline & 80000-300000 BDT per annum & 26 \\
\hline & Above 300000 BDT per annum & 1 \\
\hline \multirow[t]{2}{*}{ Previous history of TB treatment } & Yes & 97 \\
\hline & No & 3 \\
\hline History of contact with MDR-TB & & 0 \\
\hline
\end{tabular}

Table II

Drug sensitivity pattern of suspected drug resistance Tuberculosis $(n=100)$

\begin{tabular}{lcc}
\hline Drug sensitivity pattern & Number of cases & Percentage $(\%)$ \\
\hline Mono drug resistance & 19 & $19 \%$ \\
- $\quad$ Isoniazid $\quad$ Streptomycin & 13 & \\
- $\quad$ Rifampicin & 4 & \\
- $\quad$ Pyrazinamide & 2 & $18 \%$ \\
Multi drug resistance TB (MDR-TB) & 0 & $38 \%$ \\
No drug resistance & 18 & $25 \%$ \\
Culture negative & 38 & \\
\hline
\end{tabular}

Table III

Category of Patients with Drug Resistant-TB

\begin{tabular}{|c|c|c|c|}
\hline \multirow{2}{*}{$\frac{\text { Patient group }}{\text { All Relapse case }}$} & \multicolumn{2}{|c|}{ Number of patients with Multi-drug resistance } & \multirow{2}{*}{$\begin{array}{c}\text { Number of patients with Mono-drug resistance } \\
12\end{array}$} \\
\hline & After cat-I & 8 & \\
\hline & After cat-II & 2 & 2 \\
\hline \multicolumn{2}{|c|}{ Non-converter of cat-I } & 3 & 1 \\
\hline \multicolumn{2}{|c|}{ Non-converter of cat-I1 } & 1 & 0 \\
\hline \multicolumn{2}{|l|}{ Failure of cat-I } & 2 & 3 \\
\hline \multicolumn{2}{|l|}{ Failure of cat-II } & 0 & 0 \\
\hline \multicolumn{2}{|c|}{ Return after default and other } & 1(after cat- 1 ) & 1 (after cat-1) \\
\hline \multicolumn{2}{|c|}{ H/o contact with MDR TB } & 0 & 0 \\
\hline \multicolumn{2}{|c|}{ HIV and TB co-infected } & 0 & 0 \\
\hline \multicolumn{2}{|c|}{ Others $(\mathrm{H} / \mathrm{O}$ irregular treatment $)$} & 1(after cat-2) & 0 \\
\hline
\end{tabular}




\section{Discussion:}

In this study most of the affected patients belonged to 15-45 years old and male, which indicates that earning member of the family are more prone to develop drug resistance TB. This difference is because of differences in occupation, access to health care services or exposure to other risk factors. ${ }^{11}$ This trend is also observed in some other studies. ${ }^{11,12}$ In Western Europe MDR-TB cases were more common in males while, there was no association of MDRTB with the male gender in Eastern Europe. ${ }^{13,14}$ One of the underlying causes for high rate of MDR-TB among males is believed to be the reduced adherence of males to treatment compared to females and males are more exposed to external environment as they are earning member of a family. Discovering gender disparities associated with the risks of drug resistance as well as MDR-TB could provide insight into the development of targeted measures and improve health care access and reduce the risk of acquiring drug resistance.

Another important finding of our study was the higher incidence of MDR-TB among the patients below the age of 45 years $(76 \%)$. This finding has also been observed in other studies. ${ }^{14,15,16}$ Another study showed resistance to any drug as well as MDR-TB highest in 25-54 years age group. ${ }^{5}$ This is possibly due to the exposure of elderly persons to the organisms in the past, when the circulating bacilli were susceptible, and during the process of treatment resistance was acquired, while young patients are more likely to have acquired the bacilli when they were more likely to be resistant. Moreover the association between age and the risk of drug-resistance TB is not well established in the literature as different studies use different cut off point for age groups. However, to confirm the findings of this study it is important to perform a molecular epidemiological study with a larger sample size.

We found most of the affected people with drug- resistance TB live in rural area reflecting poor health care system in rural area and poor access to health services. Present study also reflects association of both drug resistance TB and MDR-TB in people with low socioeconomic condition and poor education. The possible explanation is that poor people are poor in health seeking.

In our study the variable that was more strongly associated with drug- resistance TB and MDR-TB was previous treatment as found in many other studies. The prevalence of drug resistance TB in previously treated cases was higher than new cases for each drug alone as well as for all six drugs, as reported previously. ${ }^{17,18,19}$ A national survey in china showed the rates for MDR-TB in new and previously treated cases were $5.7 \%$ and $25.6 \%$, respectively. ${ }^{17}$ In our study $19 \%$ of patients are mono-drug resistant and $18 \%$ patients have MDR-TB. ${ }^{8}$ Among MDR-TB patients all have previous history of treatment which matches with WHO findings. ${ }^{8}$ This suggests that retreatment is deficient and poses a threat to continued transmission, which has not yet manifested itself among new patients. The number of previously treated cases in the country correlate with the overall prevalence of drug resistance, as drug resistance is more commonly found in cases with previous history of antiTB medication. In another study showed patients with previous treatment of TB ranged from $4.4 \%$ to $26.9 \%$ of all registered patients under dots programmes in countries with a high burden of TB. ${ }^{20}$ In case of China and India, the two largest high-TB burden countries, $20 \%$ of sputum smearpositive cases had prior history of treatment. ${ }^{1}$ In our study among the mono-drug resistance cases $13 \%$ showed resistance to isoniazid, $4 \%$ are resistant to streptomycin and only $1 \%$ are resistant to rifampicin which is similar to the previous study. ${ }^{21}$

\section{Conclusion:}

In summary, the drug-resistance rate of PTB, especially MDR-TB, was higher in patients with previously incomplete anti-tuberculosis treatment at a tertiary referral hospital in Bangladesh. A high level of drug resistance among the retreatment TB patients poses a threat of transmission of resistant strains to susceptible persons in the community. For these reasons molecular characterization and determination of individual drug resistance of patient isolates is of importance. Proper counseling of patients and attention towards the completion of the anti-TB treatment are needed. In $\mathrm{TB}$ prevalent areas, more studies on anti-TB drug resistance preferably population based continuous surveillance should be carried out.

\section{Conflict of interest: None.}

\section{References:}

1. The World Health Organization. Global tuberculosis control: surveillance, planning, financing. WHO report 2008. Geneva WHO/HTM/TB/2008393.

2. Zignol M, Hosseini MS, Wright A, Weezenbeek CL, Nunn P, Watt CJ et al (2006) Global incidence of multidrugresistant tuberculosis. J Infect Dis 194(4):479-485.

3. Borrell S, Gagneux S. Infectiousness, reproductive fitness and evolution of drug-resistant Mycobacterium tuberculosis. Int J Tuberc Lung Dis 2009;13:1456-1466.

4. Espinal MA, Laszlo A, Simonsen L, Boulahbal F, Kim SJ, Reniero A et al. Global trends in resistance to antituberculosis drugs. World Health Organization-International Union 
against Tuberculosis and Lung Disease Working Group on Anti-Tuberculosis Drug Resistance Surveillance. N Engl J Med 2001;344(17):1294-1303.

5. Nathanson E,Lambregts-van Weezenbeek CS, Rich MJ, Gupta RJ, Bayona J, Biondal k, Caminero J.A., Cegielski PJ, Danilovits M, Espinal MA, Hollo V, JaramilloE, Leimane V,Mitnick CD, Mukherjee JS, Nunn P, Pasechnikov A, Tupasi T, Wells C, Raviglino.MC: Multidrug- resistance Tuberculosis Management in Resource- limited Settings. Emerging Infectious Disease 2006;12(9):1389-1397.

6. Espinal MA, Dye C: Can DOTS control Multidrugresistance Tuberculosis? Lancet 2005;365(9466):1206-1209.

7. Zaman K, Rahim Z, Yunus M, Arifeen S, Baqui A, et al. Drug-resistance of Mycobacterium Tuberculosis in selected urban and rural areas in Bangladesh. Scand $J$ infect dis 2005;37:21-26.

8. Multidrug or extensively drug resistant TB (MDR/XDR-TB). Global report on surveillance and response. WHO/HTM/ $\mathrm{TB} / 20103$.

9. National Guidelines and Operational Manual for Tuberculosis Control, $5^{\text {th }}$ edition, Tuberculosis Control Programme (NTP), Bangladesh. (2015).

10. Country Classification: World Bank Country and Lending Groups. Available at http://data.worldbank.org/about/ country-and-lending-groups. Accessed 16 Nov. 2016.

11. Kim S, Bai G, Hong Y. Drug-resistant tuberculosis in Korea, 1994. The International Journal of Tuberculosis and Lung Disease 1997;1:302-308.

12. Cox H, Kebede Y, Allamuratova S, Ismailov G, Davletmuratova $Z$, et al. Tuberculosis recurrence and mortality after successful treatment: impact of drug resistance. PLoS medicine 2006;3:e384.
13. Chan ED, Iseman MD. Current medical treatment for tuberculosis. Bmj 2002;325:1282-1286.

14. Faustini A, Hall AJ, Perucci CA. Risk factors for multidrug resistant tuberculosis in Europe: a systematic review. Thorax 2006;61:158-163.

15. Djuretic T, Herbert J, Drobniewski F, Yates M, Smith EG, et al. Antibiotic resistant tuberculosis in the United Kingdom: 1993-1999. Thorax 2002;57:477-482.

16. Kordy FN, Al-Thawadi S, Alrajhi AA. Drug resistance patterns of Mycobacterium tuberculosis in Riyadh, Saudi Arabia. Int J Tuberc Lung Dis 2002;8:1007-1011.

17. Zhao Y, Xu S, Wang L, Chin DP, Wang S, Jiang G, Xia H, Zhou Y, Li Q, Ou X: National survey of drug-resistant tuberculosis in China. N Engl J Med 2012;366(23):21612170 .

18. Yu C-C, Chang C-Y, Liu C-E, Shih L-F, Hsiao J-H, Chen C$\mathrm{H}$ : Drug resistance pattern of "mycobacterium tuberculosis" complex at a Medical Center in Central Taiwan, 2003-2007. J Microbiol Immunol Infect 2010;43(4):285-290.

19. Shao Y, Yang D, Xu W, Lu W, Song H, Dai Y, Shen H, Wang J: Epidemiology of anti-tuberculosis drug resistance in a chinese population: current situation and challenges ahead. BMC Public Health 2011;11:110.

20. Saltini C. Chemotherapy and diagnosis of tuberculosis. Respiratory medicine 2006;100:2085-2097.

21. M. Arévalo, J. Solera, D. Cebrian, J. Bartolomé and P. Robles, "Risk Factors Associated with Drug-Resistant Mycobacterium tuberculosis in Castilla-la-Mancha (Spain)," European Respiratory Journal 1996;9(2): 274-278. 